Revue internationale P.M.E.

Économie et gestion de la petite et moyenne entreprise

\title{
Louis-Jacques Filion, Oser intraprendre : douze modèles exemplaires, Montréal, Presses HEC, 2010, 405 p.
}

\section{Louise Cadieux}

Volume 24, numéro 1, 2011

URI : https://id.erudit.org/iderudit/1012556ar

DOI : https://doi.org/10.7202/1012556ar

Aller au sommaire du numéro

Éditeur(s)

Presses de l’Université du Québec

ISSN

0776-5436 (imprimé)

1918-9699 (numérique)

Découvrir la revue

Citer ce compte rendu

Cadieux, L. (2011). Compte rendu de [Louis-Jacques Filion, Oser intraprendre :

douze modèles exemplaires, Montréal, Presses HEC, 2010, 405 p.] Revue

internationale P.M.E., 24(1), 195-196. https://doi.org/10.7202/1012556ar d'utilisation que vous pouvez consulter en ligne.

https://apropos.erudit.org/fr/usagers/politique-dutilisation/ 


\section{Comptes rendus}

\section{OSER INTRAPRENDRE: DOUZE MODĖLES EXEMPLAIRES Louis-Jacques Filion Montréal, Presses HEC, 2010, 405 p.}

L'intrapreneuriat est un sujet de plus en plus prisé par les chercheurs en entrepreneuriat. Partant du principe que le phénomène se retrouve dans tous types d'organisation, cet ouvrage dirigé par Louis-Jacques Filion et auquel ont participé neuf auteurs brosse un portrait détaillé de onze hommes et une femme qui, au fil des ans, se sont démarqués dans leur milieu d'affaires respectif. Ce qui nous permet, avec un certain recul, de les qualifier d'intrapreneurs, notamment à cause des comportements proactifs qu'ils ont naturellement adoptés dans les différentes sphères d'activité dans lesquelles ils ont évolué ou évoluent toujours. Dans cet ouvrage, les douze cas ont été classés en trois catégories: les agents de changement et les leaders de la transformation des organisations; les créateurs de produits/services/entreprises à structures organisationnelles internes favorisant l'expression de l'innovation; et les intrapreneurs sociaux.

Dans une logique pédagogique, chaque chapitre de cet ouvrage est présenté dans le même ordre. Ainsi, après avoir introduit et résumé le cas, le lecteur est informé des objectifs d'apprentissage du cas et des questions pouvant l'aider à mieux cibler les leçons à retenir. Au même titre qu'il est invité à faire des lectures préparatoires pour l'aider à mieux comprendre l'étude de cas qui suit. Ensuite, les parcours des protagonistes sont racontés dans le détail, partant souvent de leur tendre enfance et se terminant au moment des entrevues. Qu'ont en commun les personnages auxquels on s'attache au fil des lectures? À mon grand étonnement, beaucoup! Par exemple, malgré les différentes classes sociales dont ils sont issus, la majorité d'entre eux ont grandi dans des familles où les valeurs étaient claires et, à quelques occasions, proches de celles que nous qualifions aujourd'hui d'entrepreneuriales. Dans cet environnement familial bien encadré, certains se sont, bien sûr, rebellés notamment pendant certaines périodes de leur vie où cela est normal de le faire. Toutefois, force est d'admettre que, souvent, bien malgré eux, cela a eu un impact sur leur parcours professionnel respectif. Une scolarité plus élevée que la moyenne, la maîtrise d'au moins deux langues, les voyages ou les longs séjours à l'étranger, la chance d'avoir occupé des postes de responsabilités dans de grandes organisations, le besoin de se démarquer, le besoin de s'investir avec passion dans leur travail sont d'autres points communs soulevés au fil des chapitres. 
Comme l'écrit Louis-Jacques Filion, les parcours de vie de ces douze «champions» sont inspirants. Assez pour lui avoir permis de proposer dix règles favorisant une attitude innovante lesquelles varient sur un spectre allant de la capacité de maintenir vivante une culture intrapreneuriale, à savoir aller au fond des choses, à celle d'inspirer confiance ou de bien s'entourer. Au reste, malgré le sentiment de m'être familiarisée avec le concept d'intrapreneuriat grâce aux parcours de vie de douze personnes hors du commun, je dois admettre être restée sur mon appétit, en particulier concernant les plus petites organisations ou, comme le soulevait Carrier en 1997, il serait moins probable de trouver de tels «champions », notamment à cause des environnements trop restreints dans lesquels ils devraient évoluer.

Louise Cadieux, DBA

Professeure en management

Membre de l'Institut de recherche sur les PME

Université du Québec à Trois-Rivieres

\section{Bibliographie}

CARrier, C. (1997), De la créativité à l'intrapreneuriat, Québec, Les Presses de l’Université du Québec, coll. «Entrepreneuriat et PME». 